\title{
Band Anticrossing in Highly Mismatched Compound Semiconductor Alloys
}

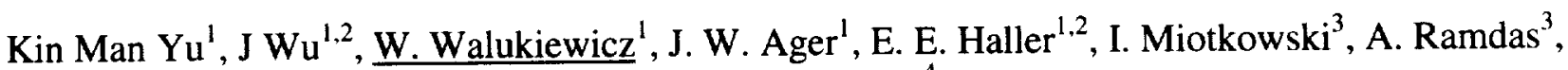 \\ and Ching-Hua Su${ }^{4}$
}

${ }^{1}$ Materials Sciences Division, Lawrence Berkeley National Laboratory, Berkeley, CA 94720

${ }^{2}$ Department of Materials Sciences and Mineral Engineering, University of California, Berkeley, California 94720

${ }^{3}$ Department of Physics, Purdue University, West Lafayette, Indiana 47907

${ }^{4}$ SD47 Science Directorate, NASA/Marshall Space Flight Center,

Huntsville, Alabama 35812

Compound semiconductor alloys in which metallic anions are partially replaced with more electronegative isoelectronic atoms have recently attracted significant attention. Group IIIN $_{\mathrm{x}} \mathrm{V}_{\mathrm{l}-\mathrm{x}}$ alloys with a small amount of the electronegative $\mathrm{N}$ substituting more metallic column $\mathrm{V}$ elements has been the most extensively studied class of such Highly Mismatched Alloys (HMAs). We have shown that many of the unusual properties of the $\operatorname{IIN}_{\mathrm{x}} \mathrm{V}_{1-\mathrm{x}}$ alloys can be well explained by the Band Anticrossing (BAC) model that describes the electronic structure in terms of an interaction between highly localized levels of substitutional $\mathrm{N}$ and the extended states of the host semiconductor matrix [1]. Most recently the BAC model has been also used to explain similar modifications of the electronic band structure observed in Te-rich $\mathrm{ZnS}_{\mathrm{x}} \mathrm{Te}_{1-\mathrm{x}}$ and $\mathrm{ZnSe}_{\mathrm{y}} \mathrm{Te}_{1-\mathrm{y}}$ alloys [2].

To date studies of HMAs have been limited to materials with relatively small concentrations of highly electronegative atoms. Here we report investigations of the electronic structure of $\mathrm{ZnSe}_{\mathrm{y}} \mathrm{Te}_{1-\mathrm{y}}$ alloys in the entire composition range, $0 \leq \mathrm{y} \leq 1$. The samples used in this study are bulk $\mathrm{ZnSe}_{\mathrm{y}} \mathrm{Te}_{1-\mathrm{y}}$ crystals grown by either a modified Bridgman method or by physical vapor transport. Photomodulated reflection (PR) spectroscopy was used to measure the composition dependence of optical transitions from the valence band edge and from the spin-orbit split off band to the conduction band. The pressure dependence of the band gap was measured using optical absorption in a diamond anvil cell. We find that the energy of the spin-orbit split off valence band edge does not depend on composition and is located at about $3 \mathrm{eV}$ below the conduction band edge of $\mathrm{ZnSe}$. On the Te-rich side the pressure and the composition dependence of the optical transitions are well explained by the BAC model which describes the downward shift of the conduction band edge in terms of the interaction between localized Se states and the conduction band. On the other hand we show that the large band gap reduction observed on the Se-rich side of the alloy system is a result of an interaction between the localized Te level and the valence bands. This interaction leads to the formation of a Te-like valence band edge that strongly interacts with the light hole valence band. Calculations based on a modified $\mathrm{kp}$ model account for the reduction of the band gap and the large increase of the spin-orbit splitting observed in Se-rich $\mathrm{ZnSe}_{\mathrm{y}} \mathrm{Te}_{1-\mathrm{y}}$ alloys. We will also discuss the importance of these new results for understanding of the electronic structure and band offsets in other highly mismatched alloy systems.

\section{References}

[1] W. Shan, W. Walukiewicz, J.W. Ager III, E.E. Haller, J.F. Geisz, D.J. Friedman, J.M. Olson, and S.R. Kurz, Phys. Rev. Lett. 82, 1221 (1999).

[2] W. Walukiewicz W. Shan, K. M. Yu, J. W. Ager III, E.E. Haller, I. Miotkowski, M. J. Seong,

H. Alawadhi and A. K. Ramdas, Phys. Rev. Lett., 85, 1552 (2000) 